\title{
Kernos
}

Revue internationale et pluridisciplinaire de religion grecque antique

$29 \mid 2016$

Varia

\section{Fabriquer du divin. Constructions et ajustements de la représentation des dieux dans l'Antiquité}

\section{Francesco Massa}

\section{(2) OpenEdition \\ Journals}

Édition électronique

URL : http://journals.openedition.org/kernos/2459

DOI : 10.4000/kernos.2459

ISSN : 2034-7871

Éditeur

Centre international d'étude de la religion grecque antique

Édition imprimée

Date de publication : 1 octobre 2016

Pagination : 478-481

ISSN : 0776-3824

Référence électronique

Francesco Massa, « Fabriquer du divin. Constructions et ajustements de la représentation des dieux dans l'Antiquité », Kernos [En ligne], 29 | 2016, mis en ligne le 01 octobre 2016, consulté le 17 novembre 2020. URL : http://journals.openedition.org/kernos/2459; DOI : https://doi.org/10.4000/kernos.2459

Ce document a été généré automatiquement le 17 novembre 2020.

Kernos 


\title{
Fabriquer du divin. Constructions et ajustements de la représentation des dieux dans l'Antiquité
}

\author{
Francesco Massa
}

\section{RÉFÉRENCE}

Nicole BELAYCHE, Vinciane PIRENNE-DELfoRGE (éd.), Fabriquer du divin. Constructions et ajustements de la représentation des dieux dans l'Antiquité, Liège, Presses Universitaires de Liège, 2015.1 vol. $16 \times 24 \mathrm{~cm}, 239$ p., $22 \mathrm{pl}$. (Religions. Comparatisme - Histoire Anthropologie). ISBN : 978-2-87562-071-2.

1 Le livre que l'on recense ici est issu du colloque parisien «Fabriquer du divin. Constructions et dynamismes de ses représentations " (INHA, les 15-17 mars 2012). Néanmoins, la publication représente également la réflexion conclusive d'un groupe de recherche européen, financé par le Conseil National de la Recherche Scientifique français et piloté par Nicole Belayche : «FIGVRA. La représentation du divin dans les mondes grecs et romains ». Il n'est pas simple de résumer en quelques lignes la richesse des activités organisées: entre 2008 et 2012, quinze rencontres scientifiques entre Paris, Rennes, Toulouse, Erfurt, Genève, Liège et Athènes, une dizaine d'ouvrages collectifs ou dossiers dans des revues scientifiques. La représentation du divin a été abordée dans des perspectives variées: les pratiques magiques, les représentations figurées, les rituels sacrificiels, les émotions, les dieux des autres, sans oublier la réflexion sur la tradition historiographique. Parler de FIGVRA signifie évoquer la fondation d'une communauté scientifique, composée de spécialistes de domaines différents, qui pendant cinq ans s'est réunie et confrontée, en constituant un lieu d'échange et de formation, notamment pour celles et ceux qui étaient des doctorant.e.s ou de jeunes docteur.e.s, et qui faisaient leurs premiers pas dans le monde de la 
recherche. C'est pourquoi il est important de situer le présent volume dans l'ensemble de la production scientifique du groupe de recherche.

2 Les onze articles qui composent le volume portent, en particulier, sur les dynamiques de la construction de la représentation des dieux ; c'est la plasticité des sociétés des dieux qui est au cœur du volume. Dans leur introduction, les éditrices soulignent que le livre vise à éclairer les processus de construction, de modification et de réélaboration de la représentation du divin : il s'agit d'une question qui est d'autant plus importante que les religions des mondes grec et romain n'avaient pas un système fondé sur une révélation ou sur des dogmes. Parmi les questionnements proposés à la réflexion par les éditrices, j'en souligne notamment deux qui me paraissent novateurs. Tout d'abord une question de méthode qui propose une réflexion sur le rapport entre "genre littéraire » (même si la catégorie est discutée) et représentation du divin : les discours littéraires sont en effet modelés différemment selon les formes littéraires utilisées; c'est pourquoi, il convient de croiser l'analyse des représentations divines avec les stratégies narratives et les pragmatiques énonciatives des sources. Ensuite, un questionnement sur les sources qui enquête sur la manière dont le rituel est en mesure de transformer l'image du dieu: c'est une perspective fondamentale qui permet de réfléchir sur les rituels comme producteurs de réflexions théologiques et pas seulement comme source d'information sur la divinité.

3 Le livre s'organise autour de trois thématiques principales. La première se concentre sur les traditions mythiques et les images et s'ouvre sur deux articles rédigés à quatre mains, ce qui est un bon exemple du travail d'équipe qui a animé le programme FIGVRA. Corinne Bonnet et Iwo Slobodzianek conduisent le lecteur dans le monde mésopotamien, pour éclairer les mythes et les rites lités à la déesse Inanna. Ils étudient en particulier deux textes, le mythe d'Inanna et Enki dans la version transmise au début $\mathrm{du} \mathrm{II}^{\mathrm{e}}$ millénaire et les Lamentations, des poèmes sumériens de la fin du $\mathrm{III}^{\mathrm{e}}$ millénaire. Leur analyse permet de comprendre comment se construisent les pouvoirs divins de la déesse Inanna et comment ces pouvoirs ont un impact sur la vie quotidienne des hommes. En combinant lecture des textes et des images, Gabriella Pironti et Vinciane Pirenne-Delforge ramènent le lecteur à la Grèce classique et aux relations divines à l'intérieur des panthéons des cités grecques. Les A. enquêtent sur les rapports entre Héra et les fils de Zeus en montrant que le conflit n'est pas la seule dimension qui émerge de l'analyse des sources. Les images sur les vases attiques, par exemple, peuvent représenter une Héra calme et bienveillante qui assiste à la naissance d'Athéna ou de Dionysos : textes et images ont leurs codes propres et indépendants, ce qui est également une importante leçon de méthode. Le langage figuré est au centre de l'analyse d'Anne-Françoise Jaccottet sur la valeur du bain du nouveau-né dans les mythes d'Achille, Dionysos et Jésus et sur le rôle des images dans le processus de création de l'identité divine. L'A. montre que le bain du nouveau-né est un observatoire privilégié pour comprendre le fonctionnement des images des dieux, leur polysémie et leurs interprétations différentes selon les contextes : dans les cas de Dionysos et Jésus, par exemple, la représentation du bain sert à souligner la double nature, humaine et divine, des deux figures.

La deuxième section du volume se concentre sur les spéculations érudites et les dynamiques visuelles dans plusieurs contextes et sources du monde romain. Les études permettent de montrer comment chaque typologie de sources (archéologiques, littéraires, iconographiques, épigraphiques) apporte un regard spécifique qui permet 
de composer un tableau plus large de la représentation du divin à Rome. Jörg Rüpke se concentre sur le rôle du prêtre romain dans la construction du divin. En passant du domaine des récits mythiques à celui des agents cultuels, il s'interroge sur le statut de sacerdotes dans les sources latines entre la fin de la République et le début de l'Empire. Il s'oppose à l'idée que les prêtres romains représentent leur divinités respectives et il montre que leur participation à la construction du divin passe davantage par leur connaissance spécifique sur les questions religieuses, notamment dans les rapports qu'ils entretiennent avec les magistrats de la ville. En restant dans la même période de l'histoire romaine, John Scheid revient sur la question du rapport entre pratique et spéculation religieuses, par le biais des dossiers des sacerdoces et cultes archaïsants et des Jeux séculaires, restaurés par Octavien/Auguste. L'A. montre l'apport des érudits dans la (re)construction des pratiques rituelles et dans la définition de nouvelles divinités. Sylvia Estienne attire l'attention sur un autre aspect de la religion romaine, la fonction religieuse des processions, souvent sous-estimée dans la bibliographie récente. Son but est notamment d'éclairer l'influence des processions dans la figuration des dieux de Rome. L'article analyse plus particulièrement l'évolution de la pompa circensis au début du Principat qui connaît l'introduction des figures de César et des princes julio-claudiens. La section est close par l'étude d'Olivier de Cazanove et François Fouriaux, qui se concentrent sur le rapport entre structures architecturales et représentation du divin, analysant comment la scénographie des monuments peut rendre manifeste la «majesté divine». Les deux exemples étudiés sont ceux est du sanctuaire du Cigognier à Avenches et des temples de l'Italie d'époque républicaine, étroitement liés à des théâtres: les auteurs mettent en évidence que la conception architecturale de ces théâtres-temples vise à présentifier la divinité lors du spectacle, " derrière et au-dessus des hommes ".

5 La troisième section s'appuie davantage sur des sources littéraires des mondes grec et romain, analysées dans leur dynamique pragmatique et performative. Les articles réunis dans cette dernière section du volume mettent en évidence l'importance de la réflexion sur les " genres » littéraires soulignée dans l'introduction. Le théâtre, lieu par excellente de la représentation visuelle des dieux, est analysé par Pierre Brulé, qui conduit le lecteur dans le monde de la représentation théâtrale euripidéenne où les divinités se montraient au public athénien ex machina. L'A. étudie les modes de représentation des dieux d'Euripide et essaie de définir comment le public voyait, entendait et écoutait la personnification divine sur scène. L'analyse détaillée (et structuraliste) de trois scènes, l'Athéna de l'Erechthée, les Discoures de l'Hélène et l'Athéna de l'Iphigénie en Tauride, permet à l'A. d'établir un tableau des résolutions divines. En passant de l'Athènes classique à la société grecque de l'époque impériale, Nicole Belayche utilise les hymnes d'époque impériale pour éclairer le type de portrait de la divinité issu de ces performances poétiques. L'A. met évidence le caractère conservateur de ces compositions poétiques qui visent au consensus politique et se fondent sur un savoir partagé par les habitants des cités de l'Empire. Toujours en contexte hymnique mais à une époque plus tardive, Gianfranco Agosti explore la religiosité du néoplatonisme du $\mathrm{v}^{\mathrm{e}}$ siècle en montrant bien que les Hymnes de Proclus s'inscrivent parfaitement dans la poésie grecque de l'époque et laissent entrevoir un dialogue et une compétition avec la production poétique chrétienne de l'époque. La troisième section se termine avec l'article de Jean-Daniel Dubois qui aborde un sujet souvent négligé par l'historiographie moderne, à savoir la pratique rituelle des communautés valentiniennes. À travers l'analyse des sources hérésiologiques et des 
textes coptes de Nag Hammadi, l'A. essaie de montrer que le rituel du baptême a joué un rôle dans la construction de la figure pléromatique de Sophia. Le livre se clôt avec un index, très utile, et plusieurs planches en couleur de bonne qualité qui facilitent la lecture et la compréhension des articles.

6 Le volume propose un parcours riche et stimulant dans les processus de construction de la représentation du divin. En analysant textes, images et pratiques rituelles, il présente une réflexion renouvelée sur les modalités de la représentation des divinités dans les mondes antiques. En s'appuyant sur une base méthodologique et théorique claire, les études soulignent le dynamisme de la représentation du divin qui s'adapte aux contextes performatifs et rituels. Je soulignerais un dernier élément fécond pour l'étude des religions et des sociétés antiques: tout au long des études, émerge une question transversale qui interroge le problème de(s) l'autorité(s) dans la fabrique du divin des mondes antiques. Dans des cultes sans révélation et sans dogmes, où se situe l'autorité ? Encore une fois, on constate que l'absence d'une autorité théologique hiérarchique oblige à tenir compte d'une pluralité de facteurs : à côté du rôle politique de la cité, le divin se construit et se représente également par les choix des poètes, des orateurs et des peintres, par les réflexions des érudits ou par la construction de l'espace sacré.

\section{AUTEURS}

\section{FRANCESCO MASSA}

Université de Genève 